\title{
Risk Spatial Distribution and Fluctuation Mechanism of Ship Traffic System Based on Catastrophe Control and Intelligent Sensor
}

\author{
Ji Qi $(\mathbb{D}$, Yuying Yuan, Wei Li, Fangjing Zhang, and Yali Li \\ College of Engineering Technical, Jilin Agricultural University, Changchun 130118, Jilin, China \\ Correspondence should be addressed to Ji Qi; jiq@jlau.edu.cn
}

Received 24 November 2021; Revised 29 December 2021; Accepted 16 January 2022; Published 24 February 2022

Academic Editor: Deepak Kumar Jain

Copyright (c) 2022 Ji Qi et al. This is an open access article distributed under the Creative Commons Attribution License, which permits unrestricted use, distribution, and reproduction in any medium, provided the original work is properly cited.

\begin{abstract}
Navigation control system is an important navigation building of inland-river in China. Because of its special semiclosed structure, when the efficiency of the mutation control system is low, the ship system cannot identify the ship, which may endanger the life safety of the crew and cause water traffic accidents. Based on catastrophe control and intelligent sensor, this paper studies the spatial distribution and fluctuation mechanism of risk in the ship traffic system, constructs a catastrophe control model, and combines with intelligent sensors to identify the spatial distribution and fluctuation of risk in ship traffic. In this paper, a typical navigation control system of inland-river is selected as the research object, and the three-dimensional calculation model of the ship traffic system is established. The numerical simulation of catastrophe control and intelligent sensor in ship traffic systems under different ventilation conditions and the navigation water level is carried out by using computational fluid dynamics method, and the spatial distribution and fluctuation mechanism of ship traffic systems are analyzed. The results show that the maximum variation of sensitivity decreases monotonously with the decrease of the plate angle. When the other design parameters of the sensor remain unchanged, the maximum variation of sensitivity reaches the minimum when the plate angle is $10^{\circ}$ which indicates that a small plate angle is helpful to reduce the eccentricity error. When the pitch increases from $56.32 \mathrm{~mm}$ to $98 \mathrm{~mm}$, the maximum change of sensitivity decreases fastest, and the decrease trend of the maximum change of sensitivity slows down. In the range of $380 \mathrm{~mm}$ pitch, the maximum change of sensitivity decreases monotonously with the increase of pitch, which indicates that increasing the properly pitch is helpful to reduce the eccentricity error.
\end{abstract}

\section{Introduction}

With the rapid development of the economy and society, the demand for water transportation has grown rapidly in the past ten years. In order to realize the observation and browsing of data in an intuitive and interactive way, we can mine and discover useful information from a large amount of complex data and discover hidden features, anomalies, associations, and laws in the data in time. We use smart sensor technology to manage the ship's transportation system. With the support of intelligent sensor technology, intelligent traffic mutation control can excavate the energy saving potential of ships from ship navigation and daily operation and also provide better traffic management scheme suggestions for managers. With the help of traffic assessment, managers can find traffic benchmarks, set up traffic benchmark levels, and learn energy-saving experiences from other ships with good traffic performance on the same route and ship type.

In the progress of the sensor, Lun et al. proposed a selfpowered chemical sensor based on graphene/semiconductor heterojunction. The photovoltaic response of the device is mainly driven by the built-in potential in the graphene based heterojunction. The built-in potential depends on the difference of Fermi levels between the two. Based on the transverse photovoltaic effect of reduced graphene oxide (RGO) film, Shi WM constructed a simple self-powered position sensor [1]. Tally prepared a kind of RGO film with a reduction gradient by reducing graphene oxide film with hydroiodic vapor at low temperature [2]. Due to the different 
reduction degrees, heterojunction will be formed between the surface layer and the inner layer. The RGO film and two symmetrical silver electrodes form a metal semiconductor metal structure, in which the Schottky contact is formed between the silver electrode and the RGO. Yang et al. used the whole empirical mode decomposition method to study the mutation diagnosis of wind turbine bearing and based on the improved density peak clustering method to diagnose the mutation of aeroengine [3]. Chang et al. tried to establish a BP neural network model to evaluate the seakeeping performance of oil tankers in strong waves and verified the feasibility of this method through seakeeping factors [4].

Meng et al. first used radial basis function neural network to estimate the wind load coefficient of ships sailing in wind waves, trained by Fourier characteristics of ship ellipse contour and three groups of ship wind load data obtained from the test, and compared the results with the experimental data, which has good consistency [5]. Vejvar et al. are the first to introduce evidence theory into ship comprehensive safety evaluation, which proves that this method can solve the uncertain decision-making problem in ship comprehensive safety evaluation [6]. Shi et al. first introduced a Bayesian network to evaluate the navigation safety of timber ships in strong waves, analyzed the influencing factors from the perspective of ship characteristics, cargo characteristics, and transportation routes, and verified the main factors inducing accidents of timber ships in strong waves [7]. Sorescu et al., by changing the course and speed of ships sailing in strong waves, discuss the motion response of ships in strong waves when the parameters change and evaluate the standard of dynamic stability of the container ship model [8]. Venus Lun and Quaddus analyze the longterm causality between spot freight and forward freight agreements and find that exogenous variables such as fleet size and fuel price have a significant impact on freight price volatility [9]. However, when the number of samples is small or the accuracy of sample data is low, the evaluation results obtained by neural network processing will produce certain errors. The procedure of ship sailing simulation experiments is complicated, and it is not easy to adjust the environmental parameters of some experiments. Most of the influencing factors of ship navigation safety have strong randomness, and the learning process of the network is slow. If the sample data learn to get a more complex network structure, it will have a certain impact on the accuracy of the results.

Based on sudden change control and smart sensors, research the spatial distribution and fluctuation mechanism of ship traffic system risks, construct a sudden change control model, and combine with smart sensors to identify the spatial distribution and fluctuations of ship traffic system risks. In this paper, a typical inland water navigation control system is taken as the research object, and a three-dimensional calculation model of the ship transportation system is established. The research uses interactive visual expressions to help people explore and interpret complex data so that maritime personnel can observe dynamic and real-time traffic information, the changing trend of ship traffic flow elements, and sort and analyze historical data to find patterns, Abnormal situations, etc. The computational fluid dynamics method is used to numerically simulate the sudden change control and intelligent sensors of the ship traffic system under different ventilation conditions and navigation water levels, and the spatial distribution and fluctuation mechanism of the ship traffic system are analyzed [10].

\section{Navigation Control System and Mutation Control}

2.1. Navigation Control System. As an important navigation building in inland-river, the navigation control system is a special navigation building dug for river crossing high mountains. The navigation control system can effectively shorten the navigation mileage of ships and plays a key role in unblocking river networks and improving navigation efficiency [11]. The navigation control system is used to control the part of the normal and reliable flight along the predetermined trajectory. The control system is composed of three parts: guidance and navigation system, attitude control system, power supply and distribution, and timing control system. Due to the large construction investment and complex technology of navigation control systems, the research on its design, construction, and safe operation is still in its infancy. At present, there are only a few small navigation control systems in China, such as the Wujiang River and Fuchunjiang River [12]. The national development and Reform Commission has proposed that the demonstration of the new waterway of the Three Gorges Project will be promoted, and a ship navigation control system will be set up on one side of the existing double line five-level ship lock of the Three Gorges project, so as to effectively solve the congestion of the Three Gorges Project and improve the navigation efficiency of ships in the middle and upper reaches of the Yangtze River [13]. The guide frame is shown in Figure 1:

As shown in Figure 1: In this context, with the more and more extensive distribution of navigation control system in the inland river shipping system, the water traffic safety in the navigation control system, especially the emission limit of ship exhaust in the control system, has gradually become the first problem to be considered in the design and operation of the control system [14]. Compared with highway and railway tunnels, the cross-section size of the ship navigation control system is larger. When the ship passes through the control system, it is necessary to limit the speed of navigation based on safety considerations, and it takes a longer time to pass through the control system. At the same time, the navigation control system itself is a typical semienclosed space. When the ventilation conditions are poor, the exhaust gas emitted by the ship cannot diffuse in a short time, and there are some pollutants in the exhaust gas. The accumulation of toxic and harmful components such as carbon monoxide $(\mathrm{CO})$ and nitrogen dioxide $\left(\mathrm{NO}_{2}\right)$ in the navigation control system will lead to poisoning of the crew's respiratory system and nervous system, threatening the crew's life safety, and at the same time, it may also lead to water traffic accidents, causing greater social impact [15]. At the same time, due to the lack of control standards for ship 

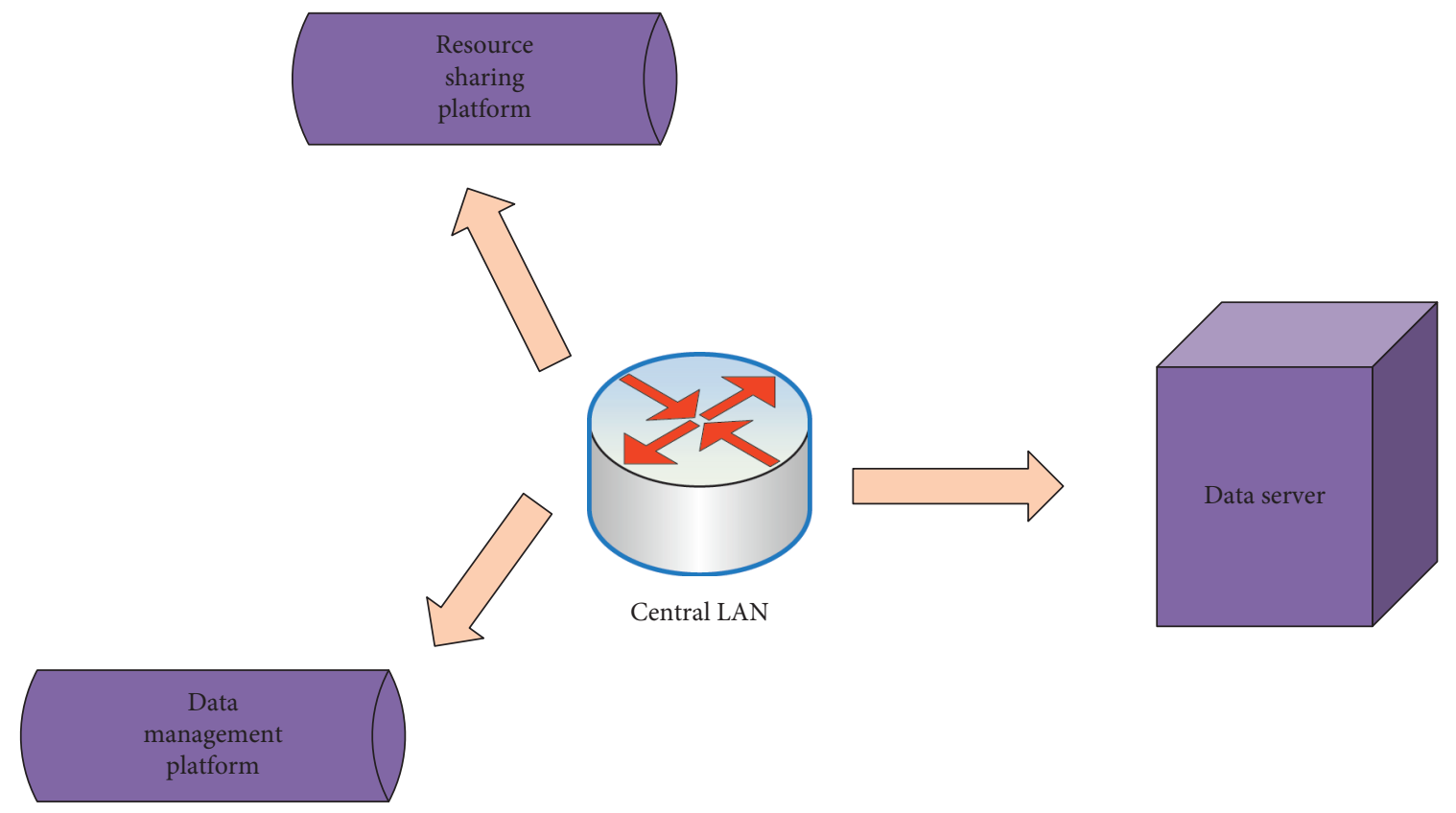

Figure 1: Navigation frame diagram.

traffic systems of the navigation control system in China, the ventilation standards for control systems such as detailed rules for ventilation design of control system are generally referred to in the design and operation of the navigation control system. However, due to the differences between the emission characteristics of ships and vehicles, the applicability of control system standards has yet to be verified [16]. Therefore, taking the typical navigation control system of the inland river as the research object, simulating the ship traffic system of the navigation control system under different ventilation conditions, and carrying out the research on the emission and diffusion of ship exhaust in the navigation control system is of great significance for ensuring the safety of water traffic and can also provide a certain scientific basis for the formulation of the design concentration standard of the ship traffic system in the navigation control system [17].

2.2. Sensor Control. With the rapid development of deep learning, artificial intelligence, the Internet of things, and other technologies, intelligent devices put forward more requirements for the increasingly complex sensor system design. For example, in a flexible electromechanical system, sensors have gradually developed from a small number of point distribution to "network" and "surface" distribution similar to a biological skin, and the number of sensors has almost increased exponentially [18]. It is a great challenge to supply energy to a large number of sensors with a wide range of distribution. Traditional sensor systems usually use a power supply (usually batteries) and cables to supply energy to sensors. When the number of sensors is small, it is easy to solve this problem. The increase of the proportion of the sensing system in the overall system restricts the further improvement of the sensing system [19]. Self-powered sensor devices provide the possibility to solve these problems. Self-powered devices can collect energy from the surrounding environment and convert it into electric energy to supply energy to their own sensor units. Therefore, they can work continuously without the support of an external power supply. When they constitute the system, the design complexity can be greatly reduced, and the distribution range and integration degree can continue to be improved [20]. In recent years, with the development of self-powered sensors and sensing systems, graphene has been widely used in self-powered sensing due to its various excellent properties [21]. Graphene can not only build sensor devices, but also capture and store energy, so it has wide application potential in self-powered sensing systems [22]. The capacitance friction electric laminated device constructed by single-layer graphene can be used as a transparent, light, and flexible self-powered tactile sensor, in which the graphene layer is not only an energy capture layer but also a tactile signal capture layer [23]. Graphene plays an important role in various types of self-powered systems, such as acting as a flexible conductive electrode, forming Schottky junction, and constructing capacitance/resistance sensor array, flow induced electricity, and so on. According to different energy supply principles or methods, graphene based self-powered sensors and systems are divided into electrochemical energy supply, photovoltaic effect energy supply, triboelectric energy supply, water volt effect energy supply, and other energy supply methods [24]. Among them, the graphene coated sponge electrode has pressure sensitive properties, and the resistance decreases with the increase of compression strain so that the voltage output of the whole zinc air battery increases with the increase of compression strain. When the battery is compressed under the maximum pressure load of $140 \mathrm{kPa}$, its output voltage remains stable and uniform in any test cycle. The strain responsive $\mathrm{Zn}$ air battery is expected to be used for self-powered pressure sensing. 
Graphene/platinum catalyzed silicone rubber (ecoflex) nanocomposites were used as electrodes by the Beijing Institute of nano-energy and systems, Chinese Academy of Sciences. Zinc wire and sodium chloride solution microcavity were combined to form a zinc air battery. The gap of graphene sheets stacked in the graphene nanocomposites expanded and the contact area decreased during the tensile process, which reflected the increasing resistance with the increase of strain. The piezoresistive effect makes the whole cell have a strain response. At $0.8 \%$ strain, the sensitivity can reach 124; when the strain reaches $150 \%$, the cell structure is not damaged. The sensor converts nonelectrical physical quantities such as temperature, pressure, liquid level, material, gas characteristics, etc., into electrical signals or sends physical quantities such as pressure, liquid level, etc., directly to the transmitter.

As shown in Figure 2, the device has a good linear response in a wide strain range of $25 \%$ to $150 \%$, and the response time is less than $0.11 \mathrm{~s}$. The sensor can be installed on the human knee joint to monitor the movement. The response of the electrochemical cell to mechanical strain not only comes from the change of electrode resistance but also from the increase of cell impedance from diaphragm when the fiber electrode is compressed. The reduced graphene oxide/carbon nanotube/sulfur fiber electrode was prepared by a simple wet spinning method in Hong Kong University of Science and Technology and assembled with lithium wire sealed in a heat shrinkable tube to prepare a wire like lithium sulfur battery. The power of the cable-shaped lithium sulfur battery fluctuates periodically between 147 and $217 \mu \mathrm{W}$ due to the increase of the diaphragm impedance. This shows that the lithium sulfur battery can be used as a fiber self-powered deformation sensor. Electrochemical cells are not only responsive to mechanical strain but also can convert humidity changes into electrical signals to become self-powered humidity sensors. For example, the lithium graphene oxide battery simply assembled from lithium foil and graphene oxide film can respond significantly to the change of humidity in the air because graphene oxide film can quickly capture the moisture from exhaled air and transfer it to the surface of lithium foil. At the same time, lithium foil is used as a microreactor to trigger the redox reaction between lithium and adsorbed water. The electrons flowing in the external circuit can reflect the respiration rate and depth in real time. Therefore, the battery can be used as a selfpowered humidity sensor to detect human respiration.

2.3. Catastrophe Control Model. The diffusion process of ship exhaust emission in the navigation catastrophe control system is greatly affected by the atmospheric flow, especially the wind speed and direction. It belongs to the unsteady three-dimensional turbulent flow, and the ship exhaust is composed of $\mathrm{CO}$ for the mixture of $\mathrm{NO}_{2}$ gas. The heat exchange of the gas is ignored in the diffusion process. Therefore, for the turbulent diffusion of the mixture, in addition to solving the momentum conservation equation and continuity equation, the corresponding turbulence control equation and component transport model should be constructed so as to accurately capture the turbulent flow of the mixture. At the same time, the validity of the CFD model should be evaluated before using the numerical simulation method to study the diffusion of the ship traffic system so as to ensure that the simulation results can truly reflect the diffusion characteristics and concentration distribution of gas.

CFD stands for Computational Fluid Dynamics. CFD is a combination of modern fluid mechanics, numerical mathematics, and computer science. It is an interdisciplinary science with strong vitality. It is to approximately express the integral and differential terms in the control equations of fluid mechanics as discrete algebraic forms, making them a set of algebraic equations, and then solving these discrete algebraic equations through a computer to obtain discrete time/space points untie.

When the Reynolds number is high, the RNGk $\varepsilon$ model is basically the same in form as the standard $k \varepsilon$ model. The main difference is that an additional generation term is added to the $\varepsilon$ equation. This item increases rapidly when the flow is rapidly distorted, thereby improving the problem of more complex turbulence such as rotating flow and buoyancy flow. In order to improve the accuracy of calculation and control efficiency of practical engineering problems, RNG K- $\varepsilon$ model is selected as turbulence control model after comparison. The two basic equations, $K$ equation and $\varepsilon$ equation, are as follows:

$$
\begin{aligned}
& k=\frac{e^{x}-e^{-x}}{e^{x}+e^{-x}}, \\
& \varepsilon=\frac{x}{1+|x|} .
\end{aligned}
$$

In this way, not only the important characteristics of the input information are preserved, but also the amount of computation can be greatly reduced. Compared with the data layer fusion, the feature layer has less data than the data layer, but the data volume is still large. Convolution neural network can adaptively extract the features of the diagnosis object and select a convolution neural network to fuse the information in the feature layer. Convolution neural network can process the original signal directly. However, the dimension of the signal collected by the vibration sensor and acoustic emission sensor is different. Even the feature extracted by a single sensor is not the same in different scales

$$
\begin{aligned}
E & =\frac{1}{2}(t-y)^{2}=\frac{1}{2}[t-f(W X)]^{2}, \\
\Delta W^{l} & =-\eta \frac{\partial E}{\partial W^{l}}=\eta\left(X^{l}\right)^{T} \delta^{l}, \\
\Delta w_{i} & =-\eta E^{\prime}=\eta x_{i}(t-y) f^{\prime}(W X)=\eta x_{i} \delta .
\end{aligned}
$$

The original signal $\Delta W^{l}$ is decomposed by wavelet packet to obtain a multisegment signal $\partial E$ with different frequency information; then the multisegment signal is processed to obtain the quantized original feature $-\eta E^{\prime}$, and the original feature is combined into a feature vector. Let $Z$ be the feature vector of a single sensor and $Z$ be the feature vector of multisensor 


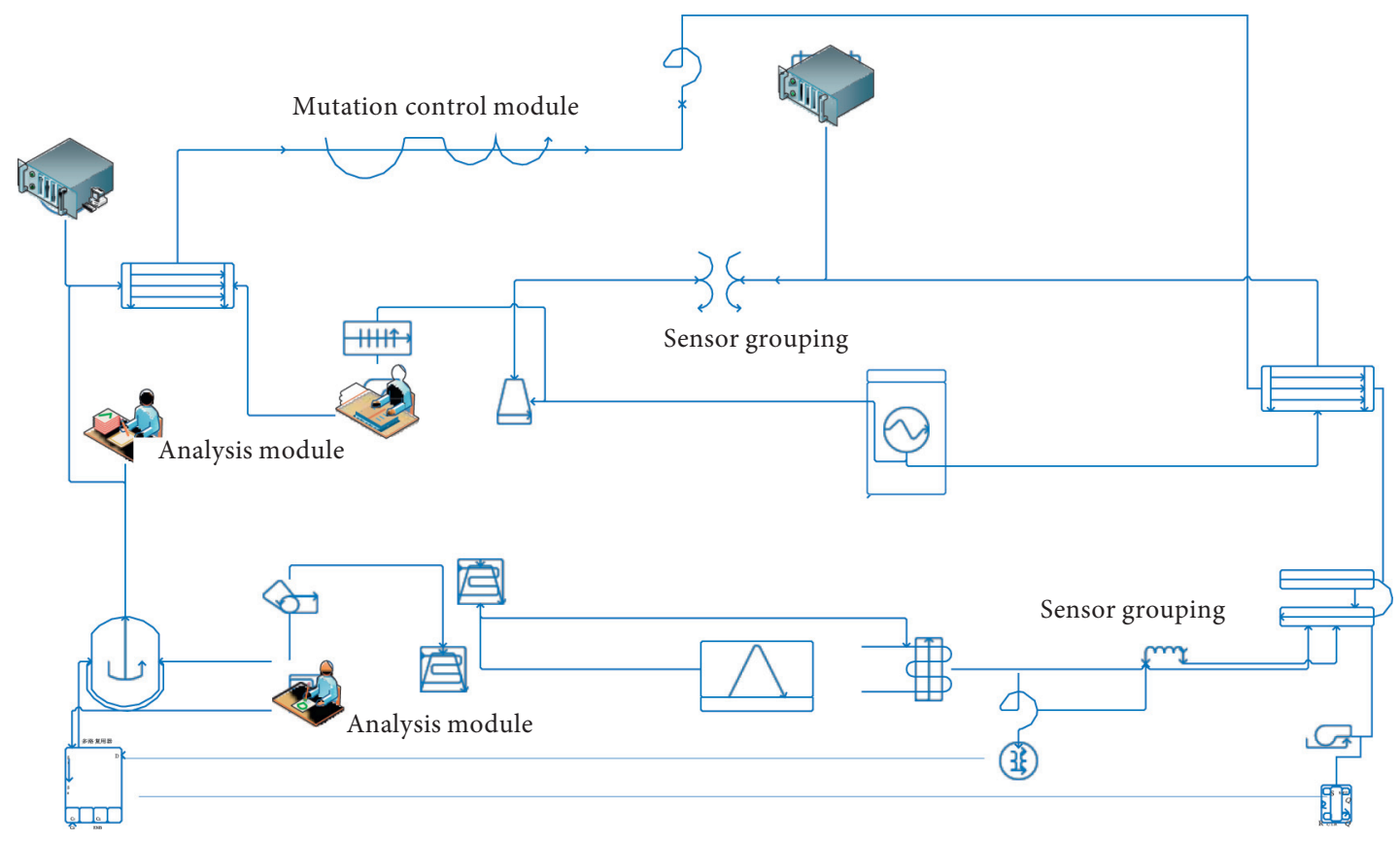

FIGURE 2: Sensor linear response relationship.

$$
\begin{aligned}
\Delta Z^{l 3} & =-\eta \delta^{l 3} X^{l 3}=\eta(t-y) f^{\prime}\left(X^{l 3} W^{l 3}\right) X^{l 3}, \\
N^{l 3} & =(t-y) f^{\prime}\left(X^{l 3} W^{l 3}\right),
\end{aligned}
$$

where $n$ is the number of extracted eigenvalues and $W$ is multiple samples. The dimension of each element in the eigenvector matrix $L$ is still not uniform, which does not conform to the data format processed by the neural network. Because the neural network focuses on capturing the differences between different types of data, and the feature vectors only compare the elements in the same position, it is necessary to convert the values of each element from absolute value to relative value to meet the needs of neural network. Let the set of elements in each column be a and the normalized transformed characteristic matrix be $n$. The relationship between $N$ and $B$ is shown in the following formula:

$$
\begin{aligned}
A_{\lim } & =f(x)=\sum_{j \subset Q} c_{j} \frac{x_{j}}{\sigma\left(X_{j}^{\prime}\right)}-p-B, \\
N & =\left(d_{i}, w_{j}\right)=P\left(d_{i}\right) P\left(w_{j} \mid d_{i}\right), \\
P\left(w_{j} \mid d_{i}\right) & =\sum_{k=1}^{k} P\left(w_{j} \mid z_{k}\right) P\left(z_{k} \mid d_{i}\right) .
\end{aligned}
$$

If the element in $P$ is $Q$, the conversion relation is as follows:

$$
Q_{\text {gain }}(Y)=\frac{\sigma(Y)-\operatorname{avg}\left(\sigma\left(Y^{\prime}\right), \sigma\left(Y^{\prime}\right)\right)}{\sigma(Y)} .
$$

The transformed eigenvalues are all greater than 0 , and the maximum value of each column element is still uncertain. It is necessary to further normalize the elements in $B$ before they can be input into the neural network. The traditional normalization method usually converts all the data to a specific interval by linear or nonlinear processing. The premise of this method is that the numerical range of the data is known, so it can be compared with each other, so it can be globally normalized. However, the data are collected by two kinds of sensors and consist of three kinds of eigenvalue arrangements. The numerical range of $\mathrm{NJ}$ in each column of data remains unchanged. Here, the value of $Q$ is uncertain. That is, the numerical range of different columns of data is different, so they cannot be compared with each other. The data processed column by column can still reflect the differences between different rows of data. Therefore, the eigenvalues are further converted to the same range by column normalization

$$
\begin{aligned}
\mathrm{Q} & =\left\{\left(a_{1}, b_{1}\right),\left(a_{2}, b_{2}\right), \ldots\left(a_{n}, b_{n}\right)\right\}, \\
\mathrm{FT} & =\arg \max \sum_{a_{n} \in W_{k}(a)} \mid\left(b_{n}=c_{n}\right) M .
\end{aligned}
$$

The element of each column in characteristic matrix $B$ is set as $m$, and the theoretical solution model of capacitive rod position sensor with double helix structure is constructed. It is generally assumed that there is no free distributed charge in the detection field space of the sensor, and the potential distribution function $\mathrm{ft}(x, y, z)$ of the detection field satisfies the Poisson equation

$$
\begin{aligned}
G(v, e)= & {\left[\zeta_{1} c_{1}(t)+\zeta_{2} c_{2}(k)+\zeta_{3} c_{3}(k)+\zeta_{4} c_{4}(k)\right.} \\
& \left.+\zeta_{5} c_{5}(k)+\zeta_{6} W_{i k}\right], \quad c_{1}(t) \geq 0, c_{2}(k) \geq 0, c_{3}(k) \\
& \geq 0, c_{4}(k) \geq 0, c_{5}(k) \geq 0,
\end{aligned}
$$

where $G$ is the relative permittivity of free space; $K$ is the distribution function of relative permittivity varying with spatial coordinates. The excitation voltage $(1 \mathrm{~s})$ of the electrode is determined by the excitation voltage $(15 \mathrm{~V})$ applied to the electrode 


$$
Q_{i k}=\sum_{a}^{n} \tau_{1} X_{i k}+\sum_{b}^{n} \tau_{2} U\left(Y_{i k}\right)+B_{i k} .
$$

The capacitance $C$ between the plates is the ratio of the amount of charge and the potential difference $u$, i.e. (4): $C=Q_{u}$ (4). Because the boundary shape of the sensor is complex, and the detection field of the capacitance sensor is a kind of "soft field," the problem can be solved effectively by the finite element method. When the capacitance of the sensor is solved by the finite element method, the physical field is established by the electrostatic element in the AC/DC module of the COMSOL program. The solving process includes the establishment of the sensor model.

\section{Navigation Control System}

3.1. Content. Based on catastrophe control and intelligent sensor, this paper studies the spatial distribution and fluctuation mechanism of risk in the ship traffic system, constructs a catastrophe control model, and combines with intelligent sensors to identify the spatial distribution and fluctuation of risk in ship traffic. In this paper, a typical navigation control system of inland-river is selected as the research object, and the three-dimensional calculation model of the ship traffic system is established. The numerical simulation of catastrophe control and intelligent sensor in ship traffic systems under different ventilation conditions and the navigation water level is carried out by using the computational fluid dynamics method, and the spatial distribution and fluctuation mechanism of ship traffic systems are analyzed.

Ship automatic identification systems are widely used in maritime traffic. A large amount of ship AIS trajectory data is collected and stored and applied to the analysis of ship traffic. AIS data usually contains multidimensional information such as time attributes, spatial attributes, and ship attributes. However, traditional two-dimensional trajectory visualization methods often only show the spatial attributes of ship trajectory data. In response to this problem, this paper proposes a three-dimensional visualization model that can simultaneously display time, space, and speed and fully demonstrate the multidimensional characteristics of AIS data. And through examples, it shows that the visual model can make people more intuitively and deeply understand the temporal and spatial characteristics of the AIS trajectory data. Finally, the ship trajectory data in the bridge area is selected for analysis and experiments, and the results show that the model can accurately represent the navigation status of the ship in the bridge area.

3.2. Design. The experimental object of this article is to select $5 \mathrm{kn}$ and $7 \mathrm{kn}$ load data when the speed is $800 \mathrm{r} / \mathrm{min}$.

The experimental design is based on catastrophe control and smart sensors to study the spatial distribution and fluctuation mechanism of ship traffic system risks and construct catastrophe control models.

In this paper, when the speed is $800 \mathrm{r} / \mathrm{min}$, the data of $5 \mathrm{kn}$ and $7 \mathrm{kn}$ load are selected as the training set data, and the load is $9 \mathrm{kn}$. When the training times reach 15 , it will reach the ideal state for the first time and then experience many ups and downs, so the training times of 25 can meet the accuracy requirements. In order to realize the intelligent diagnosis of bearing a mutation in different working conditions and types of metro traction motor, the bearing signals of unknown working conditions and mutation types are intelligently diagnosed. The output of the whole convolution layer can be regarded as a new data matrix, which contains the characteristic information of the input data. Therefore, a second convolution layer can be added for deeper feature extraction, and a pooling layer can be added between the two convolution layers for data dimension reduction. After the first convolution layer, the data is reduced from the original $40 \times 40$ to $38 \times 38$, and then through a pooling layer, because the moving step of the pooling matrix of the pooling layer is 2 , the data is converted from $38 \times 38$ matrix to $19 \times 19$ matrix, and the data dimension is reduced; then the data enters the second convolution layer, and the data is reduced from $19 \times 19$ to $17 \times 17$; then a pooling layer is added. The parameters of the pooling matrix are consistent with those of the previous pooling layer. The boundary of the pooling layer is filled with 0 , and the data is reduced from $17 \times 17$ to $9 \times 9$. At the same time, the data dimension is also greatly reduced. The full connection layer is the last part of the hidden layer of the convolutional neural network, which can get the output by a nonlinear combination of the extracted features and only transmit the signal to other full connection layers; the output layer generally uses the "softmax" classifier, which is very effective in multiclassification. The softmax classifier is modeled by the Multinomial Distribution (MultinomialDistribution), which can be divided into a variety of mutually exclusive categories. The full connection layer and the output layer can classify the samples and get the probability distribution that the current samples belong to different kinds. The experimental object of this article is to select $5 \mathrm{kn}$ and $7 \mathrm{kn}$ load data when the speed is $800 \mathrm{r} / \mathrm{min}$.

The experimental design is based on catastrophe control and smart sensors to study the spatial distribution and fluctuation mechanism of ship traffic system risks and construct catastrophe control models.

\section{Results and Discussion}

4.1. Navigation Control System. As shown in Figure 3, the ship traffic acquisition and monitoring software can collect relevant traffic data such as fuel change record, voyage mileage, arrival and departure time and name, cargo capacity, etc., within a single voyage (leg). Based on the monitoring results, it can also make statistical analysis of data and generate ship traffic data collection reports that meet the requirements of different laws and regulations. It has certain intelligent traffic management functions and has been installed and used in more than 100 ships. The trim optimization software needs to input the ship speed, draft, and other parameters in the interface to put forward the operation suggestions for the optimal trim. The purpose is to simplify the ship operation and reduce the ship operation 


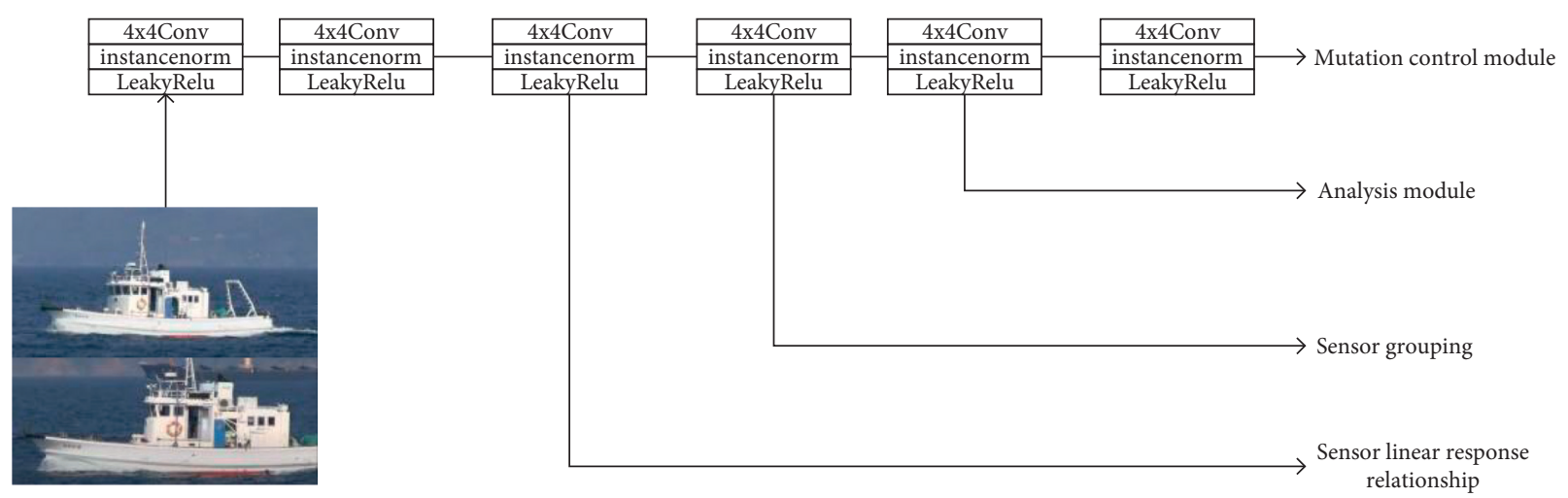

FIGURE 3: Ship energy efficiency collection and monitoring software (some pictures come from CNKI).

cost. The system has the functions of remote monitoring, fuel management, performance analysis, mutation diagnosis, trim optimization, speed optimization, and so on.

As shown in Table 1, when the test set sample is in the training set sample interval, the average diagnostic accuracy reaches $95.14 \%$; when the test set sample is greater than the upper limit of the training set sample interval, the average diagnostic accuracy reaches $90.28 \%$; when the test set sample is less than the lower limit of the training set sample interval, the average diagnostic accuracy reaches $65.28 \%$. From the diagnostic results of each sensor, the average diagnostic accuracy obtained by using information fusion technology reaches $83.57 \%$; when using vibration sensor as information source alone, the average diagnostic accuracy reaches $80.79 \%$; when using acoustic emission sensor as information source alone, the average diagnostic accuracy reaches $70.14 \%$.

Make the training set and test set contain 54 types of test data at the same time, and the diagnosis results are shown in Figure 4. In the figure, the vertical axis represents the actual mutation type of the bearing, which corresponds to six mutation types, each mutation type contains nine working conditions, and there are 54 signals of the tested samples; the horizontal axis represents the probability of neural network predicting six mutation types. The final output type is the type of the maximum row value, so when the diagnosis is correct, the column mutation type of the block where the probability value is located is the same as the row mutation type. When the information fusion technology is not used, the inner ring crack and inner ring pitting mutation types are misjudged in the diagnosis mode based on vibration sensor and acoustic emission sensor, and the diagnostic accuracy is not $100 \%$, and the overall confidence is low. After using the information fusion technology, not only the diagnostic accuracy is improved to $100 \%$, but also the confidence is higher.

As shown in Figure 5, in the structure of the sensor part, the excitation electrode is defined as the terminal and a voltage of $1 \mathrm{~V}$ is applied. The detection electrode, the measured rod and the shielding layer are grounded. At the same time, the air domain outside the sensor model is established and designated as an infinite element domain to eliminate the influence of the volume product of the air domain in the model on the calculation results. The relative permittivity of the ceramic tube is defined as 9.6.
The mesh independence of the calculation model is verified, and the mesh generation is carried out in three ways: relatively refined, ultrarefined, and extremely refined, as shown in Figure 6 . When the rod is in the position of $0 \mathrm{~mm}$, the number of domain units obtained by the above three grid generation methods is 289600, 615200, and 8292000 , respectively, and the capacitance calculated by the three grids is $312.02,308.85$, and $305.53 \mathrm{pf}$, respectively. Compared with the third grid, the relative errors of the first two grids are $2.12 \%$ and $1.08 \%$, respectively. Considering the calculation accuracy and speed, the accuracy of the first two grids is verified. It is more reasonable to use super-fine mesh.

As shown in Figure 7, during the static characteristic experiment of capacitive rod position sensor, the measured rod moves in the sensor with a certain step length, and the grating linear displacement sensor follows the measured rod and provides the true value of rod position. The rod position measurement prototype will measure the capacitance of the sensor when the measured rod is in the corresponding step number and display the capacitance detection signal through the data acquisition and processing program. Then, the measured rod position can be obtained by converting the capacitance distribution at each rod position.

It can be seen from Figure 8 that with the increase of pipe wall thickness, the maximum change of sensitivity decreases monotonously, which indicates that an appropriate increase of pipe wall thickness is helpful to reduce eccentricity error. At present, the relative permittivity of the ceramic tube is 9.6, and other design parameters of the fixed sensor remain unchanged. The maximum variation of the sensitivity decreases monotonously with the decrease of the relative permittivity of the tube wall, which indicates that the smaller relative permittivity of the tube wall is helpful to reduce the eccentricity error of the sensor.

It is assumed that the measured rod is parallel to the central axis of the sensor when it is eccentric. The calculation range of $L$ is $0 \sim 1 \mathrm{~mm}$, and the unit is $0.1 \mathrm{~mm}$. The calculation results obtained by using the above finite element model are shown in Table 2. The optimization process of capacitive rod position sensor involves four related parameters, so it is difficult to realize the full parameter combination analysis. Therefore, the orthogonal experimental design method is used to optimize the four parameters of the sensor. 
TABLe 1: Mutation diagnosis accuracy.

\begin{tabular}{|c|c|c|c|c|c|c|}
\hline Item & Rod under test & Shield & Training set & Test set & Refine & Accuracy (\%) \\
\hline Sensitivity & 1.21 & 1.26 & 1.84 & 0.73 & 0.62 & 89 \\
\hline Eccentric & 1.8 & 2.45 & 3.52 & 1.8 & 1 & 85 \\
\hline Displacement & 5.35 & 3.39 & 2.4 & 2.35 & 4.47 & 92 \\
\hline Plate & 2.39 & 2.34 & 2.57 & 5.54 & 3.69 & 93 \\
\hline Sensor & 3.27 & 1.66 & 1.27 & 1.28 & 2.93 & 95 \\
\hline Control rod & 3.93 & 2.29 & 2.28 & 2.35 & 4.05 & 98 \\
\hline
\end{tabular}

Pancare condition fault diagnosis relationship

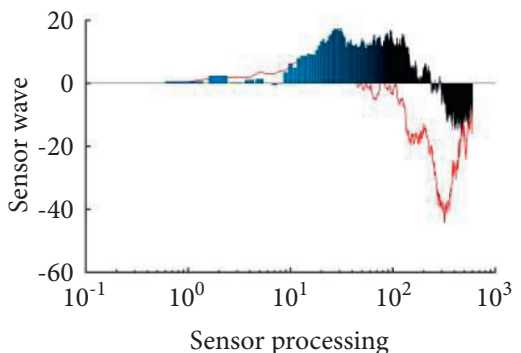

- malfunction

- Image Processing

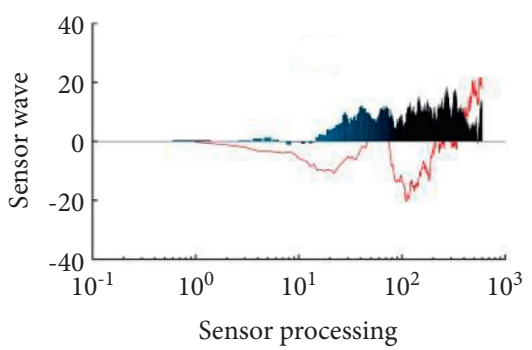

- malfunction

— Image Processing

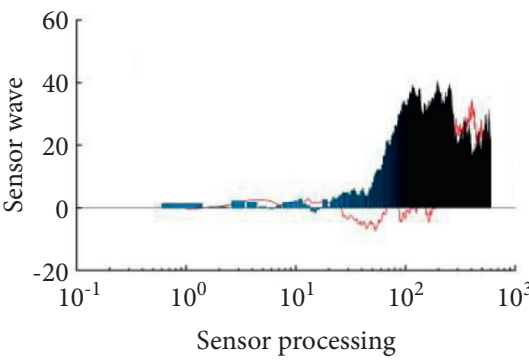

- malfunction

- Image Processing

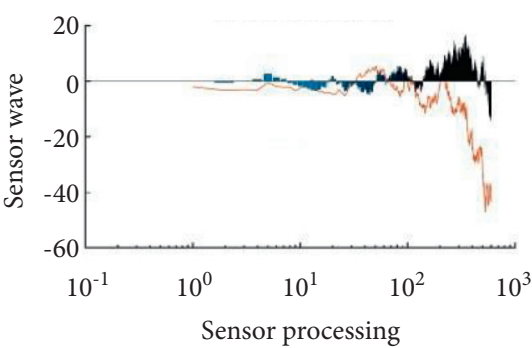

- malfunction
— Image Processing

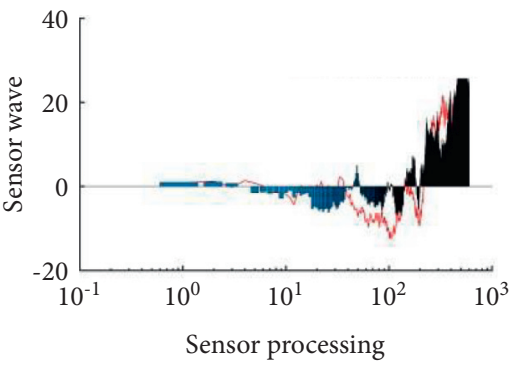

— malfunction

— Image Processing

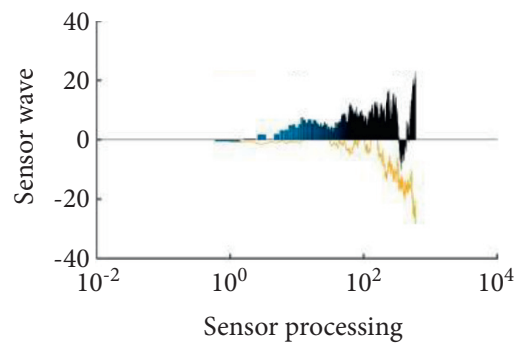

- malfunction - Image Processing

FIgURe 4: Pancare condition fault diagnosis relationship.

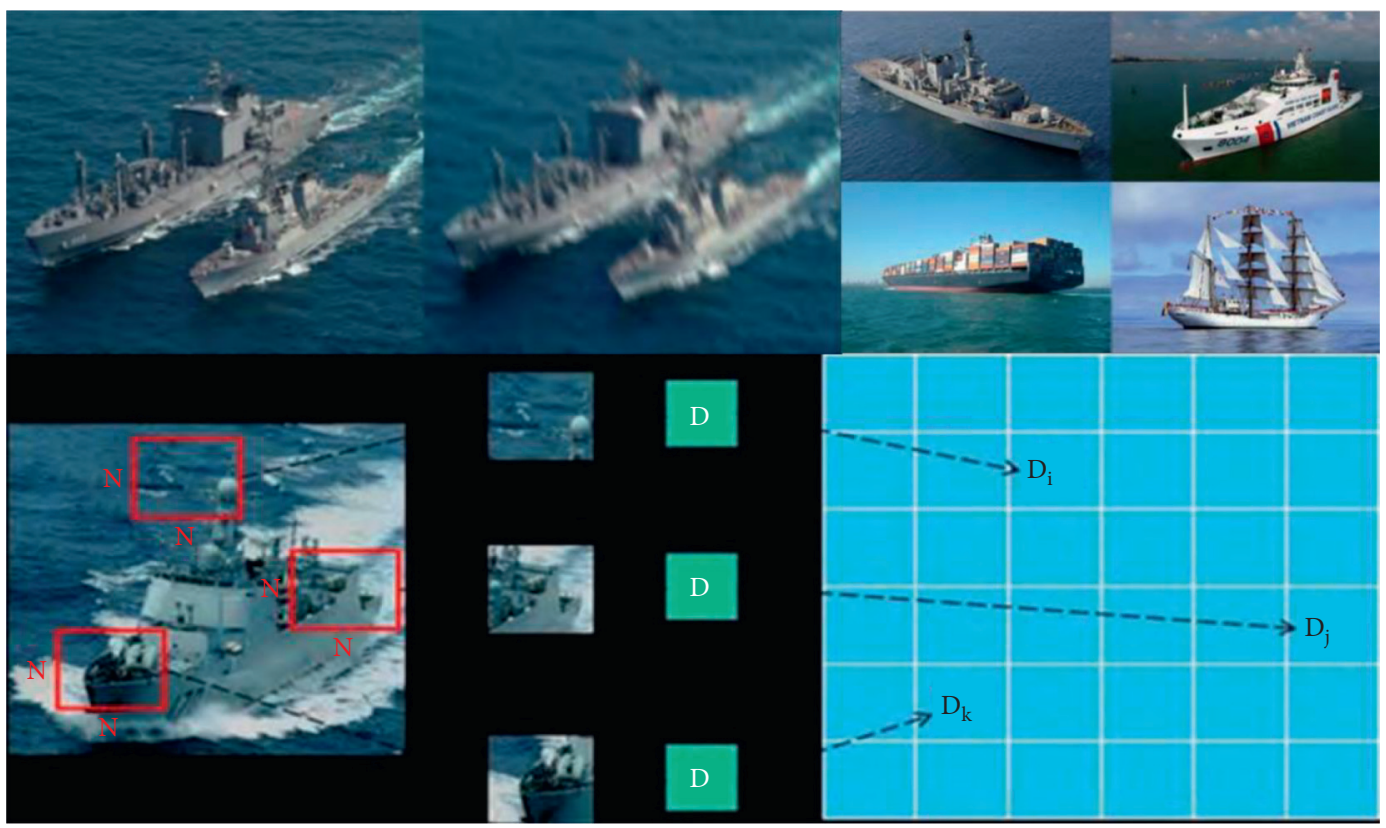

FIgURE 5: The electrode is defined as the terminal voltage (some pictures come from CNKI). 
Keywords co-occurrence overlay mapping for shipping management

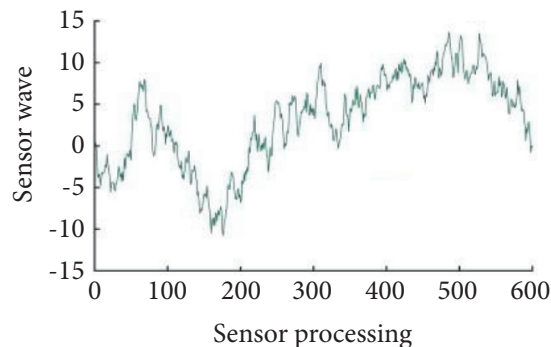

_ malfunction

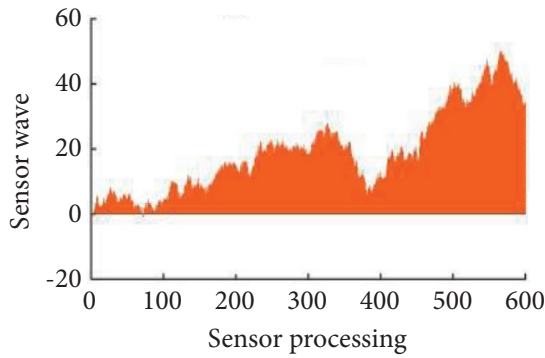

_ malfunction

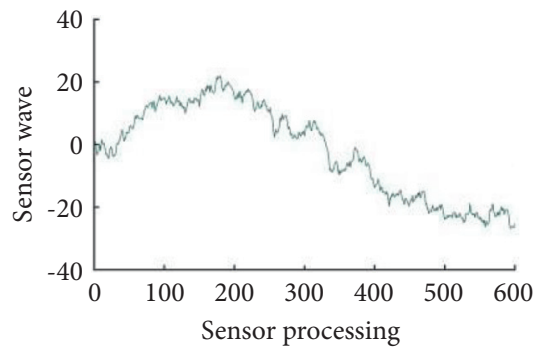

_ malfunction

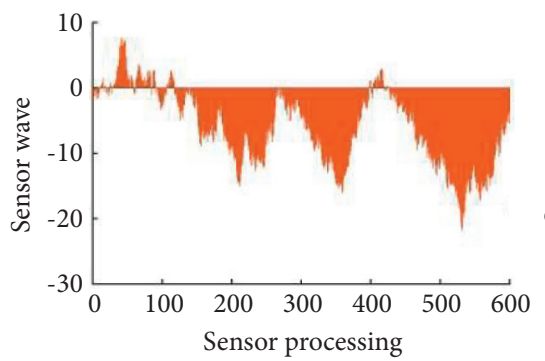

_ malfunction

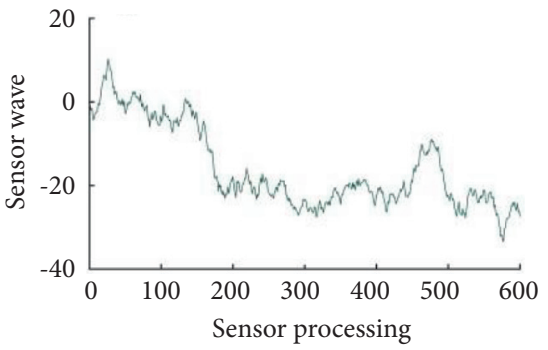

_ malfunction

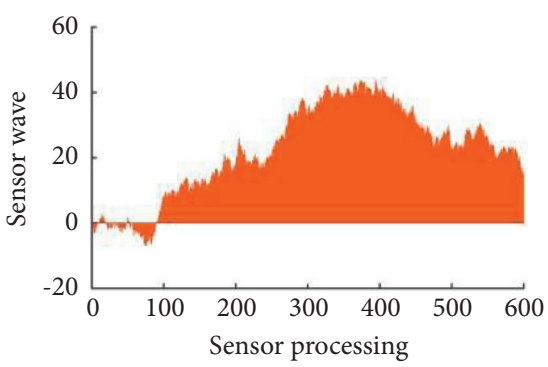

malfunction

FIGURE 6: Keywords cooccurrence overlay mapping for shipping management.

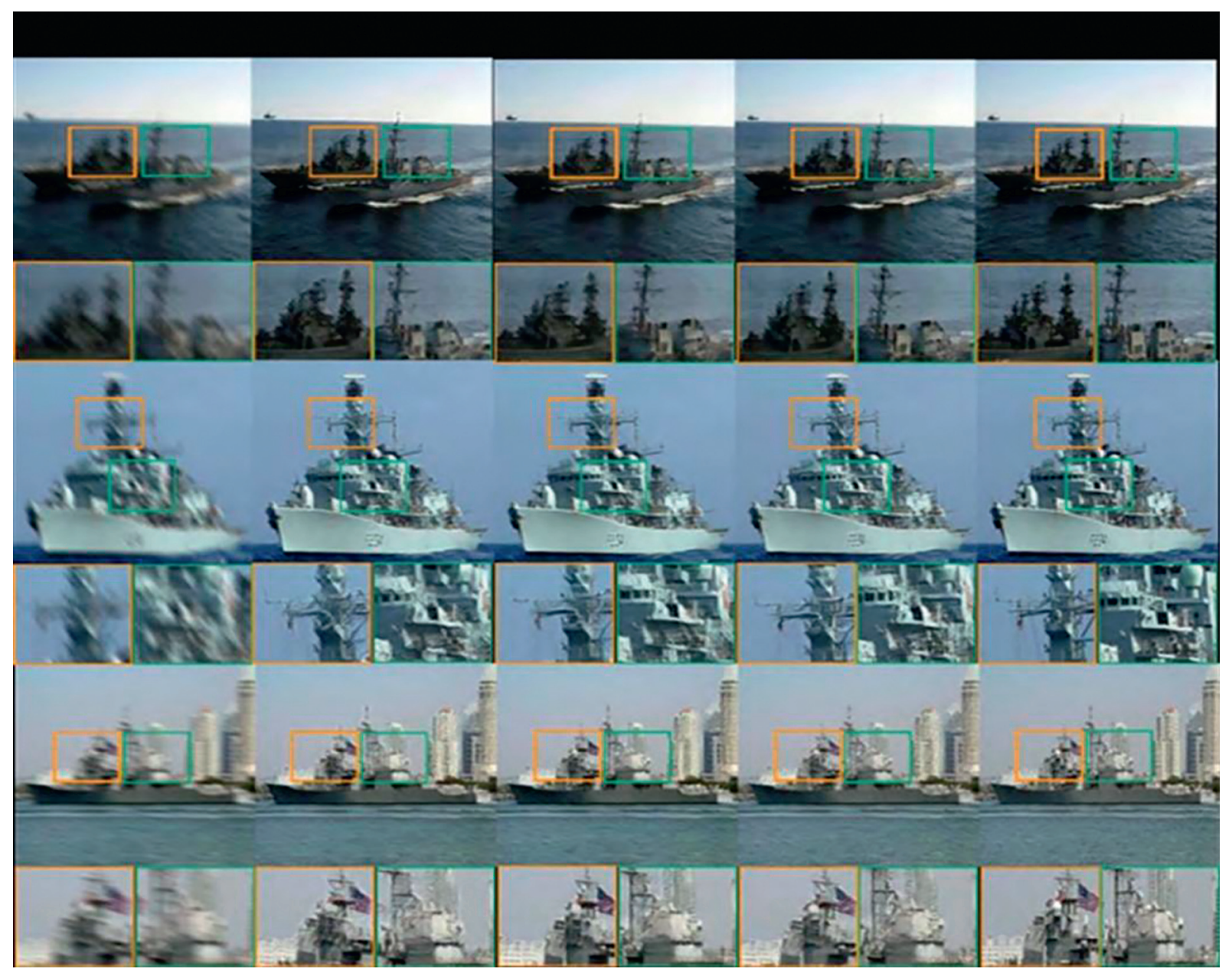

FIGURE 7: Static characteristics of the sensor (some pictures come from CNKI). 


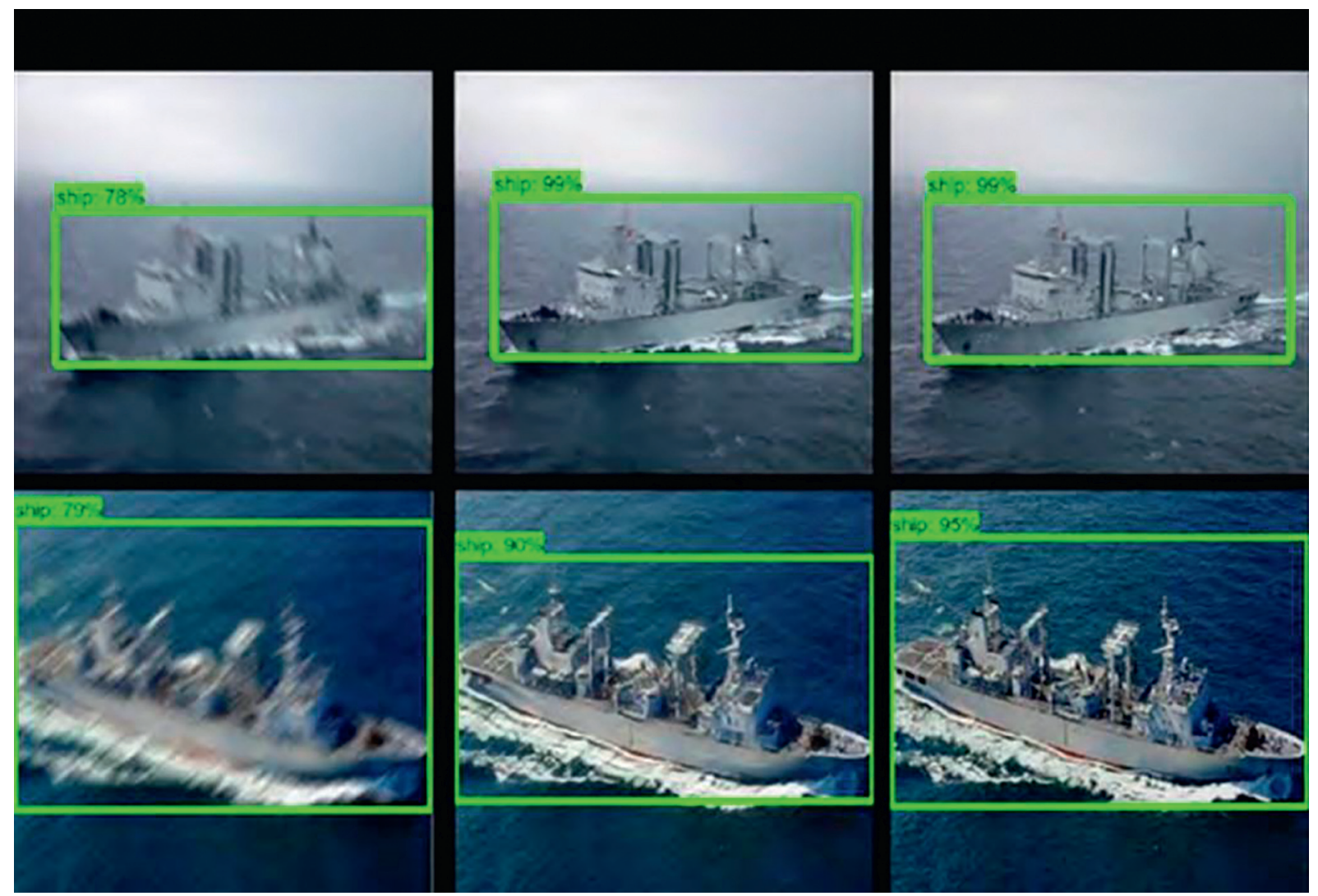

FIgURE 8: Maximum change in sensitivity (some pictures come from CNKI).

TABLE 2: Orthogonal combination optimization analysis.

\begin{tabular}{|c|c|c|c|c|c|c|}
\hline Item & Control rod & Measuring rod & Stick position & Detect & Electrode & Sensor \\
\hline Sensitivity & 1.2 & 0.12 & 0.04 & 0.42 & 0.5 & 1.26 \\
\hline Eccentric & 3.28 & 1.17 & 2.38 & 3.17 & 3.49 & 3.98 \\
\hline Displacement & 5.67 & 3.38 & 3.27 & 3.71 & 4.3 & 4.15 \\
\hline Plate & 1.15 & 5.02 & 2.21 & 2.65 & 1.57 & 1.55 \\
\hline Training set & 3.18 & 4.67 & 4.62 & 3.93 & 4.15 & 2.13 \\
\hline Test set & 4.54 & 5.33 & 2.21 & 4.77 & 1.7 & 3.31 \\
\hline
\end{tabular}

As shown in Figure 9, the absolute value of sensitivity increases with the increase of eccentric displacement $L$. when $l$ is taken as $1 \mathrm{~mm}$, the absolute value of sensitivity reaches the maximum, and when the measured rod is not eccentric, the absolute value of sensitivity is the minimum. At present, the electrode plate angle used in the sensor is $170^{\circ}$ and other design parameters of the fixed sensor remain unchanged. The influence of the electrode plate angle on the maximum change of sensitivity is analyzed, and the calculation interval is $10^{\circ}$ to $170^{\circ}$ with $20^{\circ}$ as the unit.

As shown in Table 3, with the decrease of the plate angle, the maximum variation of sensitivity decreases monotonously. While other design parameters of the sensor remain unchanged, when the plate angle is $10^{\circ}$, the maximum variation of sensitivity reaches the minimum value, which indicates that a smaller plate angle is helpful to reduce the eccentricity error. At present, the pitch $h$ of the sensor plate is $53.59 \mathrm{~mm}$, and other design parameters of the fixed sensor remain unchanged. The influence of the pitch on the maximum change of sensitivity is analyzed. The calculation range is $100 \sim 400 \mathrm{~mm}$, and the unit is $100 \mathrm{~mm}$.

As shown in Figure 10, when the pitch increases from $56.32 \mathrm{~mm}$ to $98 \mathrm{~mm}$, the maximum variation of sensitivity decreases the fastest, and then the decreasing trend of the maximum variation of sensitivity slows down. In the range of $380 \mathrm{~mm}$ pitch, the maximum change of sensitivity decreases monotonously with the increase of pitch, which indicates that increasing the pitch properly is helpful to reduce the eccentricity error. At present, the tube wall thickness of the sensor is $2.8 \mathrm{~mm}$, and other design parameters of the fixed sensor remain unchanged. The influence of the tube wall thickness on the maximum change of sensitivity is analyzed. The calculation interval is $2-3 \mathrm{~mm}$, and the unit is $1 \mathrm{~mm}$. Here, the concept of diameter pole ratio is used to control the tube wall thickness. The diameter pole ratio $\rho=R 2 / R 3$, that is, the ratio of the inner radius and the outer radius of the ceramic tube. 


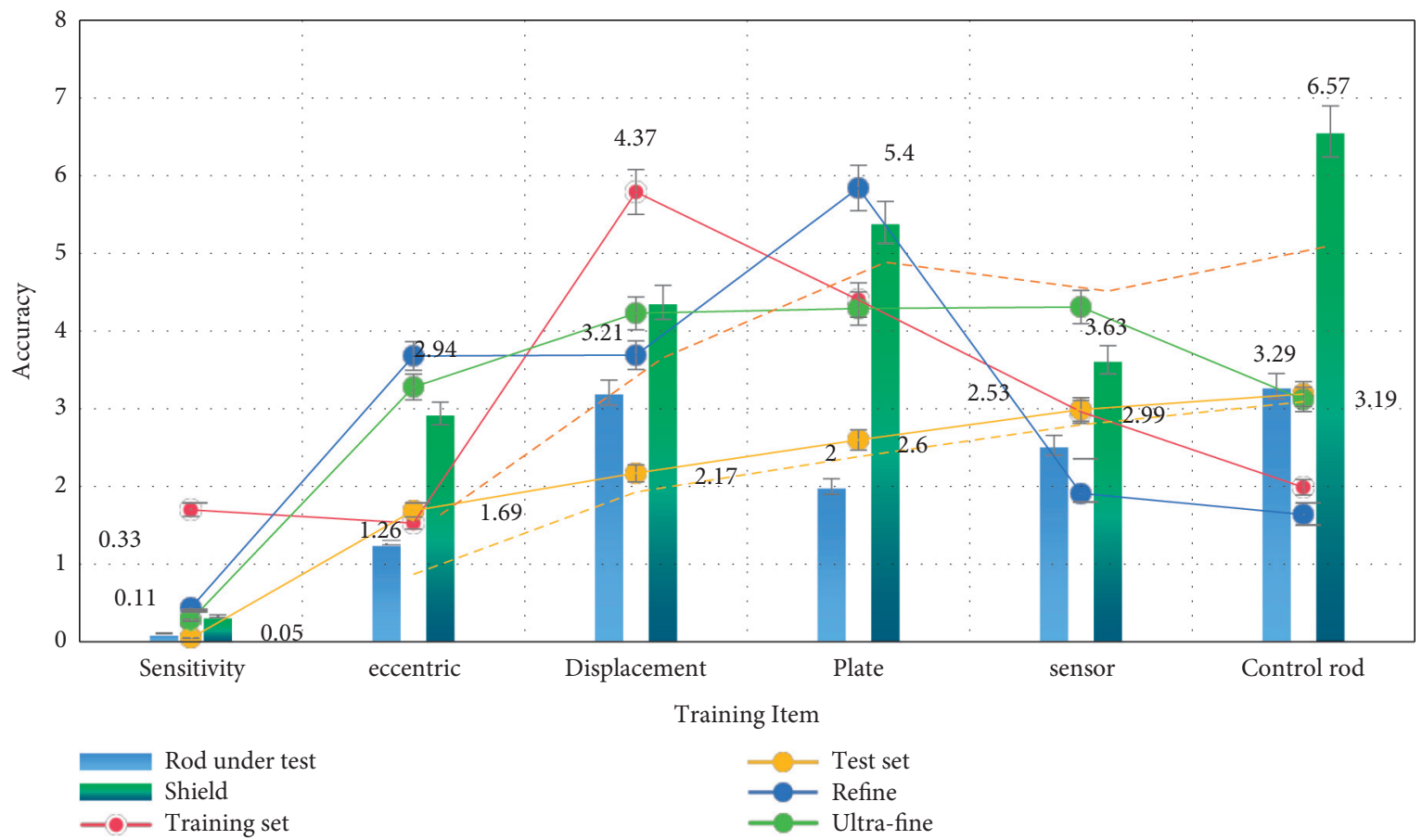

FIgURE 9: Absolute value of sensitivity varies with eccentricity.

TABLE 3: Calculation results obtained from the finite element model.

\begin{tabular}{|c|c|c|c|c|c|c|}
\hline Item & Sensitivity & Eccentric & Displacement & Plate & Training set & Test set \\
\hline Control rod & 0.02 & 1.74 & 0.25 & 1.3 & 1.15 & 1.52 \\
\hline Measuring rod & 3 & 3.81 & 2.13 & 1.35 & 1.57 & 2.87 \\
\hline Stick position & 3.26 & 5.01 & 2.8 & 5.48 & 4.56 & 3.31 \\
\hline Detect & 4.03 & 1.22 & 5.07 & 5.15 & 5.89 & 1.47 \\
\hline Electrode & 2.78 & 3.03 & 1.64 & 2.1 & 1.17 & 1.49 \\
\hline Sensor & 5.83 & 6.46 & 7 & 4.29 & 5.52 & 4.91 \\
\hline
\end{tabular}

As shown in Figure 11, the influence of speed change on signal is greater than that of load change; for the same mutation bearing, the frequency distribution and amplitude difference are obvious under different speeds and loads. As a whole, the difference caused by speed and load can make the difference overlap between different mutation types of bearings. The acoustic emission signal is similar to the vibration signal. When the bearing mutation is small or even weak, the extracted signal features cannot reflect the real situation of the mutation due to the low signal-to-noise ratio. At this time, the frequency range can be predetermined, and then the filter is used to process the signal, and finally, the feature is extracted. In this paper, there are differences in all kinds of mutation type bearings in the whole frequency band, and the conventional filtering method cannot control the differences between all kinds of mutation type bearings well.

As shown in Figure 12, by optimizing the operation management of ships, improving the traffic level of ship operation and reducing fuel consumption, it is not only beneficial for shipping enterprises to achieve cost reduction and efficiency, but also conducive to fulfilling the requirements of international emission reduction regulations. The implementation of ship traffic management is conducive to enhancing the competitiveness of shipping enterprises, which have a high demand in shipping enterprises. With the continuous development and application of information, sensing, communication, artificial intelligence, and other enabling technologies, the real ship application of ship traffic management technology is promoted.

As shown in Figure 13, ship traffic is a branch of many traffic fields. Ship traffic management is mainly for operating ships, which refers to the use of relevant technical means to strengthen the operation management in the process of ship navigation, including traffic parameter monitoring based on sensors, intelligent evaluation of traffic level, and traffic management strategy formulation based on model analysis. Generally speaking, the technology set of improving the traffic level of operating ships through operation and management innovation is called ship traffic management technology. With the development of modern information technology and artificial intelligence, the application of ship traffic management technology is promoted. On the basis of traditional management technology, intelligent algorithm and technology are applied to provide assistant decisionmaking functions for ship management and operators, 


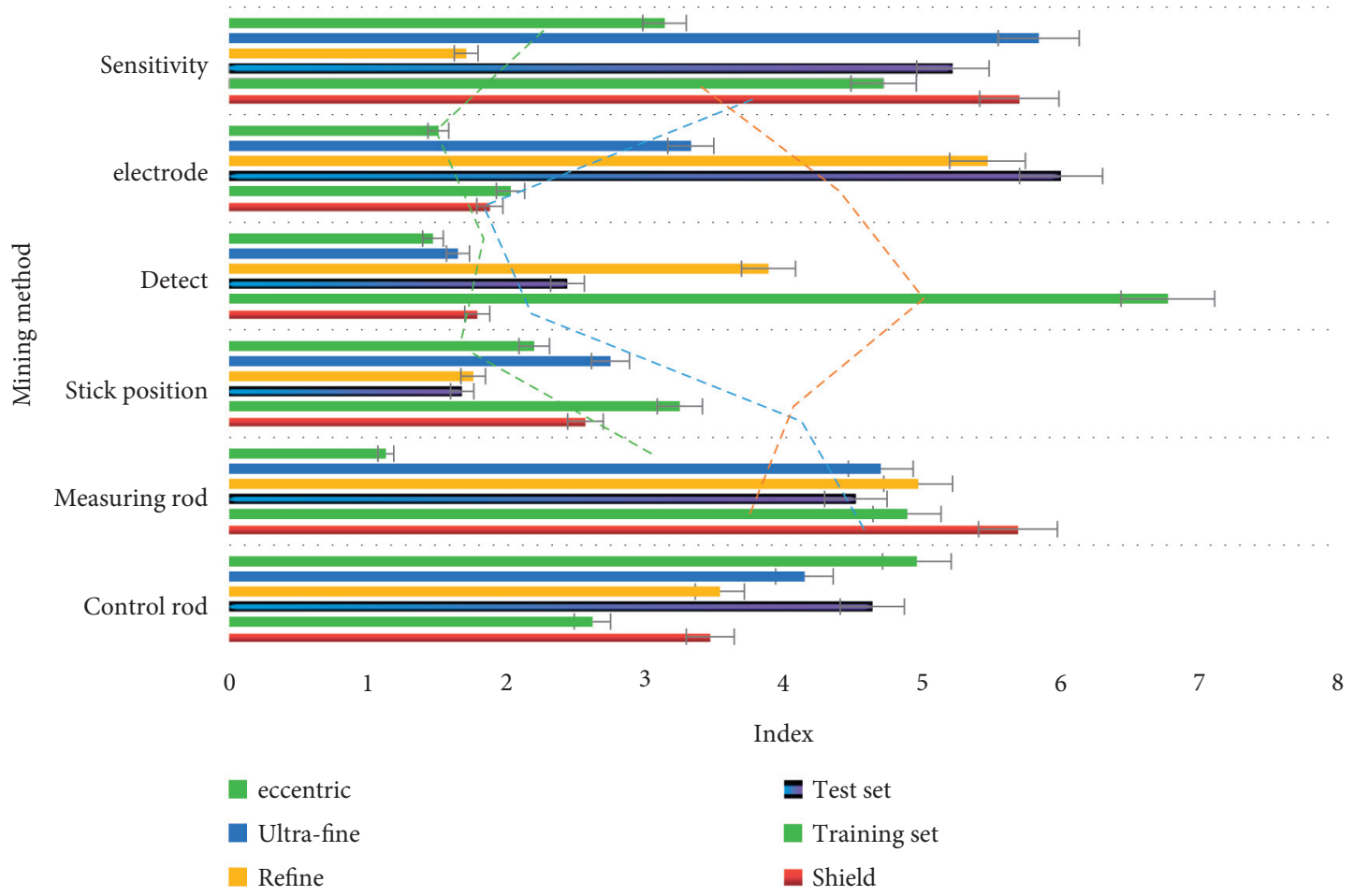

FIGURE 10: Sudden change of control sensitivity.

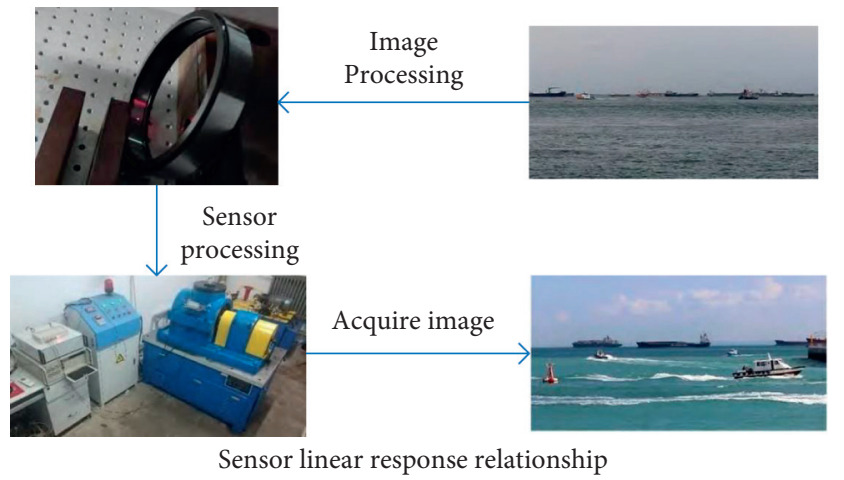

Figure 11: The influence of speed change than load change on signal (some pictures come from CNKI).

which is called intelligent traffic management technology. Taking (intelligent) traffic management technology as the core, supplemented by relevant software and hardware systems, can form (intelligent) traffic management system. After the system has a high degree of maturity and productization, it will form the related products of ship traffic management.

4.2. Discussion. The traffic management system is developed for several inland ships. Through the real-time acquisition of navigation environment data such as water depth, water flow speed, wind speed, and direction, as well as navigation attitude data such as ship speed, course, longitude, and latitude, as well as energy consumption data (engine fuel consumption, power, torque, and speed), according to the dynamic response relationship model between ship traffic and navigation environment, it can be used in different navigation environment. The developed traffic management system has been successfully applied in inland luxury cruise ships, bulk carriers, and diesel LNG dual fuel ships. Harbin Engineering University has developed the integrated traffic intelligent management system (imsmee), which has the functions of online monitoring of traffic parameters, energy consumption distribution analysis, energy consumption equipment traffic evaluation, trim, speed, route optimization, and other intelligent management functions. It has formed a relatively complete traffic management product and completed the demonstration application on two VLCC ships of the Dalian factory in 2019. Based on the big data center platform, CSIC 711 research institute has developed the product of ship traffic management system, which has been applied in navigation mark ships, steam ferries, and tugs.

Through comprehensive comparison and analysis of domestic and foreign technology development status, it can be concluded that the functions of ship traffic management system mainly include four aspects: online monitoring of ship traffic, traffic evaluation, traffic optimization, and traffic auxiliary functions. Among them, online traffic monitoring can collect traffic data and serve the other three functions, mainly monitoring the ship's energy consumption, environment, navigation, and other parameters; traffic evaluation is based on the data collected by online monitoring, using intelligent sensor technology to get the ship's energy consumption status; energy consumption optimization gives the ship the best reference value of speed, route, and trim, so as to improve the ship's traffic operation; The traffic assistance function mainly provides logs and reports that meet the requirements of IMO and maritime department. It can 


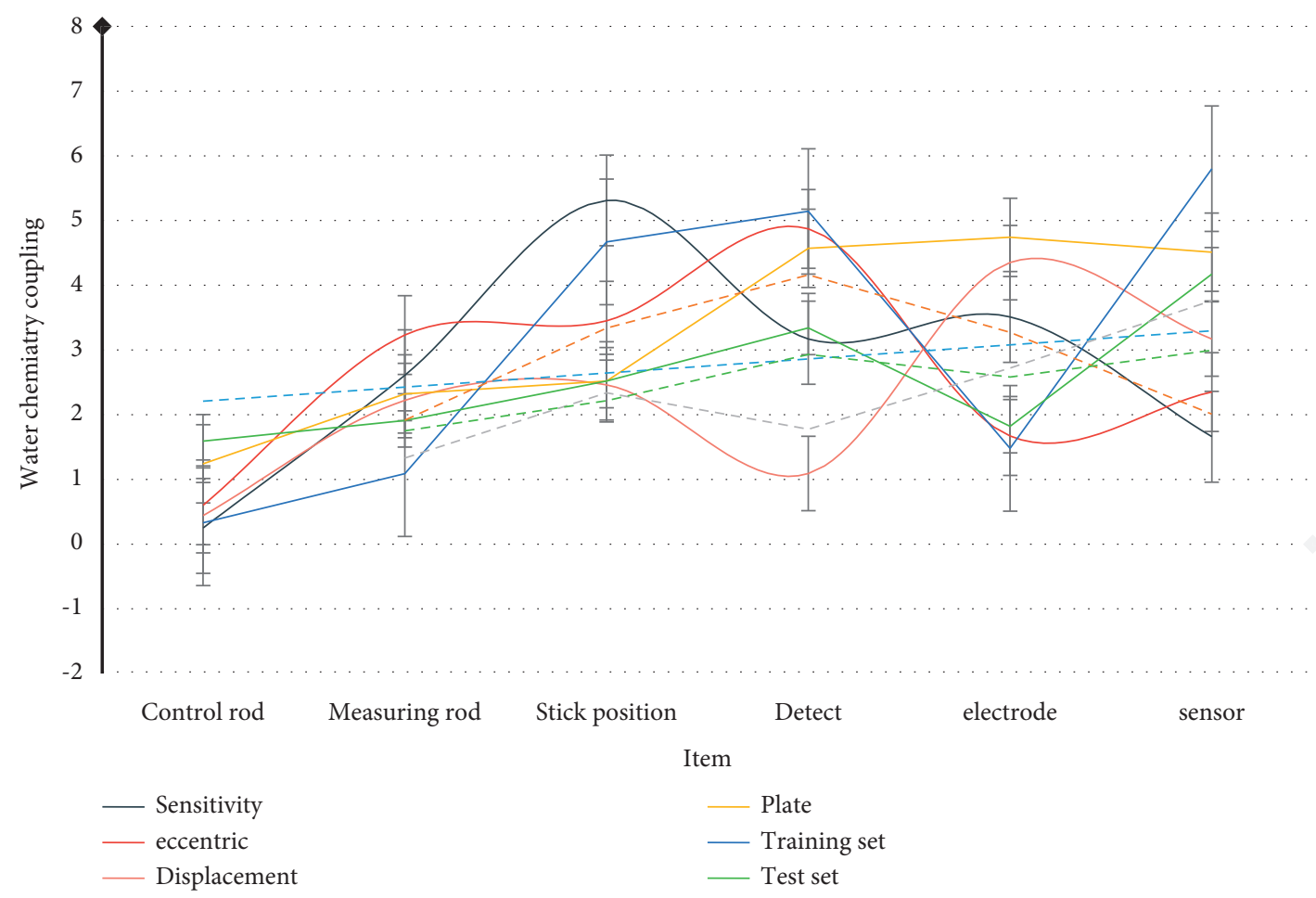

Figure 12: Optimize ship operation management.

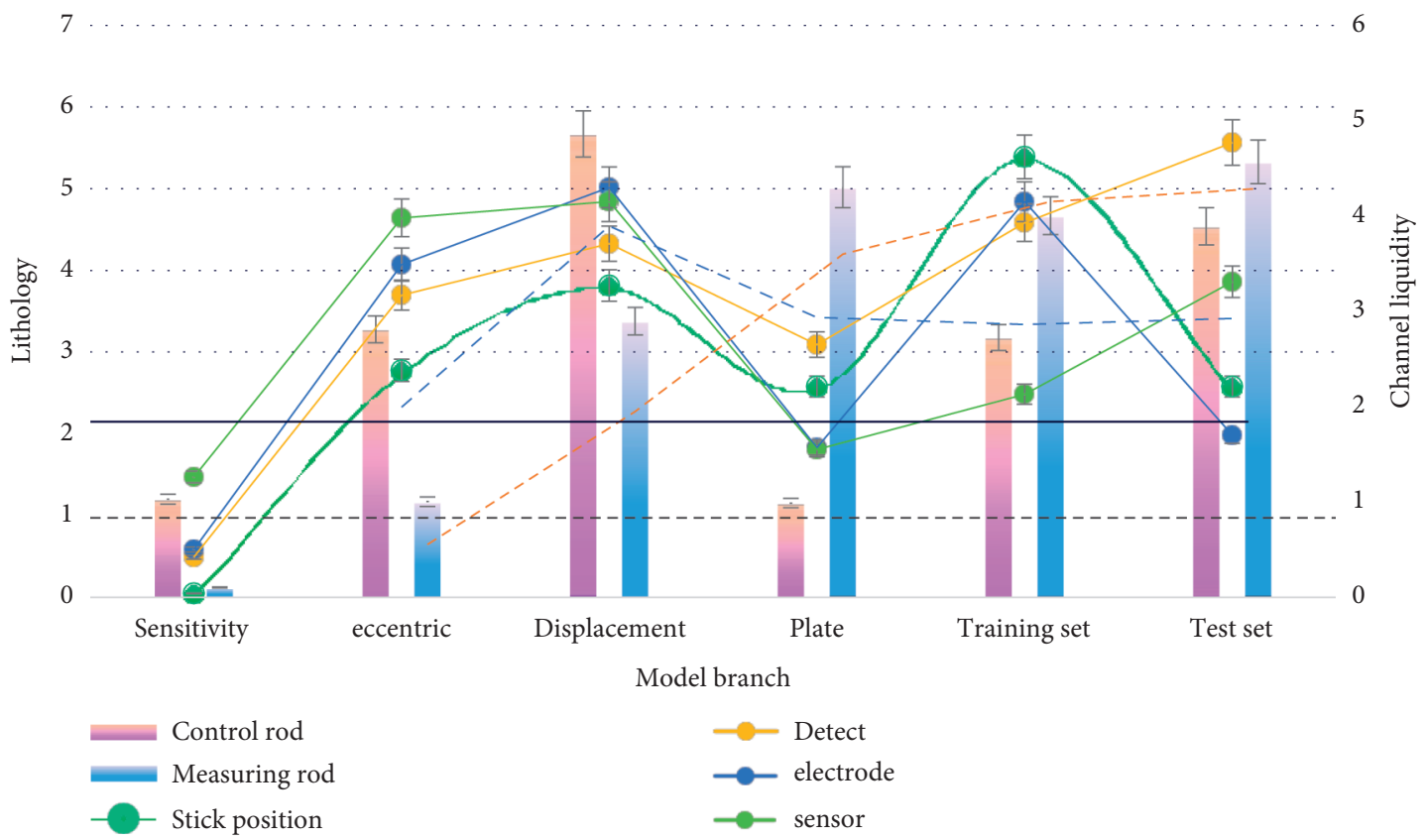

FIGURE 13: Operation management during ship navigation.

be seen that online traffic monitoring, intelligent traffic assessment, and traffic optimization control are the key technologies of ship traffic management. The change of load and speed is introduced to adapt to the diagnosis between the known condition (speed and load) samples and the unknown condition samples (or the diagnosis between the cross condition samples), and the adaptive condition diagnosis is expected.

\section{Conclusions}

At present, most of the research on ship traffic management at home and abroad is based on the monitoring of energy consumption and power or noon report data, and then gradually develop derivative functions such as traffic evaluation, speed optimization, trim optimization and mutation diagnosis, which have been applied in different ship types and 
achieved good traffic saving results. In terms of technical level, foreign institutions are relatively mature in traffic monitoring sensors and intelligent analysis software, with a higher degree of productization, occupying the mainstream market of ship traffic management at home and abroad. In the aspect of application scenarios, the research of domestic and foreign institutions in this field is multifaceted to large-scale sea going ships, while the research on inland ships with double engines and double propellers and complex hydrological environment is less. In terms of the degree of intelligence, ship traffic management at home and abroad is still based on offline analysis, while the intelligent evaluation and optimization characterized by dynamic, online, and autonomous is relatively lacking. In the future, with the promotion of modern information technology, artificial intelligence and other enabling technologies, ship traffic management will further develop towards the direction of functional intelligence and intensive management. From simple data collection and analysis, to put forward some optimization suggestions, and then to online monitoring, traffic evaluation, auxiliary optimization suggestions and other functional integration, the intelligent function is gradually enhanced. Relevant traffic data is collected at the ship end, and the data of a single ship is transmitted to the shore end; the shore end integrates the traffic data of fleet and even ships of other companies, and managers can view it in real time through the mobile client app. Specialized platform companies will provide cloud "as a service" for shipowners. Shipowners do not need to be equipped with high-performance processors on the ship and shore but only need to provide relevant data to the cloud. Due to the widespread internal conflicts, the rational pursuit system and the motivation of rational behavior are fluctuating systems. When certain conditions are met, this kind of fluctuation is within controllable range-limited fluctuations. The complex calculation and simulation are run on the cloud high-performance computer of "as a service" company, mining and analyzing massive ship traffic data, carrying out single ship or fleet traffic evaluation and optimization services.

\section{Data Availability}

No data were used to support this study.

\section{Conflicts of Interest}

The authors declare no conflicts of interest.

\section{Acknowledgments}

This work was supported by the Scientific Research Foundation of Jilin Provincial Education Department (JJKH20200333KJ), National Natural Science Foundation of China (41671397), Scientific Research Foundation of Jilin Provincial Ecological Environment Department (2019-02, 2020-18), and the Capital Construction Program Foundation within the Budget of Jilin Province Science and Technology Development Program Foundation of Jilin Province (20191001008XH).

\section{References}

[1] W. Shi and K. X. Li, "Themes and tools of maritime transport research during 2000-2014," Maritime Policy and Management, vol. 44, no. 2, pp. 151-169, 2017.

[2] W. K. Tally, "Maritime transportation research: topics and methodologies," Maritime Policy and Management, vol. 40, no. 7, pp. 709-725, 2020.

[3] Q. Yang, X. Xin-Lian, and Z. Jia-Bao, "Research status and prospect of fleet planning," Journal of Trraffic and Transportation Engineering, vol. 10, no. 4, pp. 85-90, 2020.

[4] Y. Chang, Z. Xiao-Ning, and L. Wang, "Review on integrated scheduling of container terminals," Journal of Trraffic and Transportation Engineering, vol. 19, no. 1, pp. 136-146, 2019.

[5] Q. Meng, S.-A. Wang, H. Andersson, and K. Thun, "Containership routing and scheduling in liner shipping: overview and future research directions," Transportation Science, vol. 48, no. 2, pp. 265-280, 2020.

[6] M. Vejvar, K.-H. Lai, and C. K. Y. Lo, "A Citation network analysis of sustainability development in liner shipping management: a review of the literature and policy implications," Maritime Policy and Management, vol. 47, no. 1, pp. 1-26, 2020.

[7] W. Shi, Y. Xiao, Z. Chen, H. McLaughlin, and K. X. Li, "Evolution of green shipping research: themes and methods," Maritime Policy \& Management, vol. 45, no. 7, pp. 863-876, 2018.

[8] F. Sorescu, R. Bosneagu, and C. E. Coca, "Strategic research of the maritime market," Acta Universitatis Danubius Administratio, vol. 5, no. 1, pp. 39-48, 2020.

[9] Y. H. Venus Lun and M. A. Quaddus, "An empirical model of the bulk shipping market," International Journal of Shipping and Transport Logistics, vol. 1, no. 1, pp. 37-54, 2020.

[10] Y. W. Xu, W. W. Cai, and T. C. Xie, "Fault Diagnosis of subway traction motor bearing based on information fusion under variable working conditions," Shock and Vibration, vol. 2021, Article ID 5522887, 12 pages, 2021.

[11] J. J. Xu, T. L. Yip, and P. B. Marlow, "The Dynamics between freight volatility and fleet size growth in dry bulk shipping markets," Transportation Research Part E: Logistics and Transportation Review, vol. 47, no. 6, pp. 983-991, 2020.

[12] Y. Jing-Bo, M.-F. Luo, and F. Li-Xian, "Dynamics and interactions between spot and forward freights in the dry bulk shipping market," Maritime Policy and Management, vol. 44, no. 2, pp. 271-288, 2017.

[13] R. Adland, F. E. Benth, and S. Koekebakker, "Multivariate modeling and analysis of regional ocean freight rates," Transportation Research Part E: Logistics and Transportation Review, vol. 113, no. 4, pp. 194-221, 2018.

[14] Z. H. Munim and H.-J. Schramm, "Forecasting container shipping freight rates for the far east-Northern Europe trade lane," Maritime Economics and Logistics, vol. 19, no. 1, pp. 106-125, 2017.

[15] P. Eslami, K. Jung, D. Lee, and A. Tjolleng, "Predicting tanker freight rates using parsimonious variables and a hybrid artificial neural network with an adaptive genetic algorithm," Maritime Economics \& Logistics, vol. 19, no. 3, pp. 538-550, 2017.

[16] Z. Yang and E. E. Mehmed, "Artificial neural networks in freight rate forecasting," Maritime Economics and Logistics, vol. 21, no. 3, pp. 390-414, 2019.

[17] G. F. De Oliveira, "Determinants of European freight rates: the role of market power and trade imbalance," Transportation Research Part E: Logistics and Transportation Review, vol. 62, no. 5, pp. 23-33, 2020. 
[18] P. Zi-Xuan, S. Wen-Xuan, F. Guan, and Y. Bin, "Stable vesselcargo matching in dry bulk shipping market with price game mechanism," Transportation Research Part E: Logistics and Transportation Review, vol. 95, no. 5, pp. 76-94, 2020.

[19] R. Chen, J.-X. Dong, and C.-Y. Lee, "Pricing and competition in a shipping market with waste shipments and empty container repositioning," Transportation Research Part B: Methodological, vol. 85, no. 7, pp. 32-55, 2016.

[20] D. Lei, H. Hu, C. Feier, and Z. Jianing, "The dynamics between newbuilding ship price volatility and freight volatility in dry bulk shipping market," International Journal of Shipping and Transport Logistics, vol. 7, no. 4, pp. 393-406, 2020.

[21] R. Adland and H. Y. Jia, "Shipping market integration: the case of sticky newbuilding prices," Maritime Economics and Logistics, vol. 17, no. 4, pp. 389-398, 2020.

[22] X. Bai and J. S. L. Lam, "An Integrated analysis of interrelationships within the very large gas carrier (VLGC) shipping market," Maritime Economics \& Logistics, vol. 21, no. 3, pp. 372-389, 2019.

[23] R. Agarwal and O. Ergun, "Ship scheduling and network design for cargo routing in liner shipping," Transportation Science, vol. 42, no. 2, pp. 175-196, 2020.

[24] Q. Meng and T. S. Wang, "A scenario-based dynamic programming model for multi-period liner ship fleet planning," Transportation Research Part E-Logistics and Transportation Review, vol. 47, no. 4, pp. 401-413, 2020. 